\title{
Instrumento para Medir Satisfacción de Pacientes sobre Atención Clínica Odontológica
}

\author{
Instrument to Measure Patient Satisfaction about Dental Care Clinic \\ Gonzalo Vásquez,***; Gilda Corsini**; Monica Silva**; Jorge Fuentes ${ }^{* *}$; \\ Mariana Chahin $^{* *} \&$ Juan Diego Santibáñez ${ }^{* *}$
}

VÁSQUEZ, G.; CORSINI, G.; SILVA, M. FUENTES, J.; CHAHIN, M. \& SANTIBÁÑEZ, J. D. Instrumento para medir Satisfacción de pacientes sobre atención clínica odontológica. Int. J. Odontostomat., 10(1):129-134, 2016.

RESUMEN: El objetivo fue dar a conocer la importancia de creación de un instrumento de medición de satisfacción de atención para los pacientes que concurren a las clínicas odontológicas de la Universidad de La Frontera. Los criterios utilizados tanto para la justificación de la creación como los elementos que deben conformar el instrumento de medición, fueron obtenidos de una amplia revisión bibliográfica de distintas índoles sociales, obteniendo datos sobre la importancia de registrar y procesar la satisfacción del usuario como instrumento de mejoramiento en el mundo moderno de la calidad del servicio entregado por las instituciones. Además, entrega componentes que debieran ser incorporados en una encuesta de satisfacción para el grupo objetivo seleccionado.

PALABRAS CLAVES: educación, medición, encuesta, calidad, satisfacción.

\section{INTRODUCCIÓN}

Las universidades trabajan arduamente en generar un recurso humano que satisfaga las necesidades de la población, intentando formar profesionales de calidad, con formación ética y humanista, para la realización de distintas acciones tanto en individuos como en la comunidad. En este punto, los profesionales egresados deberán incorporarse a los sistemas de salud actuales, donde las vertientes apuntan al desarrollo e integración de los sistemas de salud. Sin embargo, Schweiger \& Álvarez (2007) explican que actualmente hay carencia de métodos de medida que establezcan claramente la situación actual de los sistemas y servicios de salud en cuanto a la calidad de los servicios entregados a los usuarios.

En el contexto global de las instituciones educacionales observamos que la introducción de instrumentos de evaluaciones se ha ido incrementando, acercando más las opiniones de los usuarios de un servicio a la generación de cambios en torno a las necesidades que éstos requieren.
A su vez, actuales instrumentos y sistemas de medición presentan falencias, tal como expresa el MINSAL (2013), lo que implica que al no existir datos que aporten evidencia científica, es imposible realizar cambios en los distintos sistemas de salud, y en consecuencia, aportar datos fidedignos para ejecutar cambios. Lo anterior es aplicado a muchas organizaciones, que no contemplan opiniones evaluativas de los usuarios.

Actualmente, varias universidades e instituciones carecen de un sistema de medición de calidad de atención y satisfacción de los pacientes atendidos en éstas, que según la visión actual de las instituciones, es fundamental para establecer la problemática evidenciada desde otra mirada, la del usuario del servicio, tal como se está realizando en otros países (Tsuchida et al., 2003)

Determinantes de calidad y satisfacción de un servicio. Usuario y cliente se usan comúnmente en las

\footnotetext{
* Magister en Educación Mención Evaluación Educacional. Universidad de La Frontera, Temuco, Chile.

* Especialista en Endodoncia, Facultad de Odontología, Universidad de La Frontera, Temuco, Chile.

*** Magíster en Ciencias de a Educación, Mención Evaluación. Universidad de Concepción, Concepción, Chile.
} 
áreas de los servicios sociales y de la salud comunitaria, con la intención de erradicar la referencia a un sujeto pasivo y dependiente. En la actualidad, muchas instituciones de salud tienen la convicción de que para evaluar los servicios se requieren, sin duda, las expresiones del paciente, las cuales remiten a un ser humano que sufre el dolor o el malestar, pero que tiene derechos a la atención y a la salud.

En términos sociológicos y administrativos, paciente es el sujeto que recibe los servicios de un médico u otro profesional de la salud. Es un ser humano que, además de tener derecho a la atención y a la salud, también tiene el derecho a ausentarse de sus actividades habituales y responsabilidades, tal como lo obliga, en cumplimiento de las recomendaciones médicas, el cuidado de la enfermedad. (Velandia Salazar et al., 2007; Miranda Ortiz et al., 2014).

A diario, un sujeto acude a las instituciones de salud en espera de algo, otros sujetos lo atienden con la esperanza de poder satisfacerlo y, en caso de que estos lleguen a constatar que no le aportan lo previsto, esperan que en la próxima oportunidad, puedan arreglar las falencias y otorgar una mejor atención, tal como corrobora Velandia Salazar et al. Por su parte, Trucco (2004), Miranda Ortiz et al. y López-Portilla et al. (2013) explican que existen dos modelos para establecer calidad en las instituciones de salud. El primero consiste en la medición de calidad en función de la aprobación de los clientes y, un segundo modelo, enfocado a la prevención o alivio del sufrimiento humano causado por la enfermedad.

En las instituciones universitarias que otorgan atención variada a pacientes, los puntos anteriormente mencionados deben ser considerados a cabalidad, ya que en la actualidad el paciente no se conforma únicamente con la relación existente con el profesional, sino con lo recibido en forma integral por parte de la institución.

Así mismo, Urrutia \& Poupin (2010) y LópezPortilla et al. explican que las medidas clásicas utilizadas en salud no son suficientes para evaluar la calidad de los servicios otorgados. En la actualidad y en los países desarrollados, la evaluación de la calidad de atención es un parámetro fundamental a considerar al momento de evaluar un servicio de salud, donde la opinión del usuario es un indicador prioritario.

Los conceptos primordiales en el mejoramiento actual de los distintos servicios de distintas institucio- nes involucran necesariamente calidad de atención y satisfacción del usuario englobando, no sólo la relación profesional con el paciente (López-Portilla et al.).

Massip Pérez et al. (2008) y Miranda Ortiz et al. definen el concepto de calidad en la atención médica como las distintas actividades que garantizan un servicio de salud accesible y equitativo con profesionales sumamente buenos y teniendo en cuenta los recursos disponibles para lograr la satisfacción del usuario con la atención recibida. Trucco dice que la calidad es fundamental en los servicios de salud y que la calidad de la atención en salud, entendida como un enfoque centrado en el cliente, es una de las estrategias predominantes en la gestión de salud actual y explica que ésta consiste en la realización de los valores positivos de todo tipo y la evitación de los negativos.

Respecto a lo anterior, la medición de la calidad de la atención debe ser cuantificada por parte del usuario, quien debe decidir si la percepción del servicio recibido fue buena o mala. Miranda Ortiz et al. y Velandia Salazar et al., reafirman lo anterior, y explican que el usuario estima lo bueno o lo malo de las creencias, de la percepción y de la cognición de éste. A su vez, este autor establece dos dimensiones en lo que respecta a los estándares de calidad: i) Cuando se habla de una dimensión macro se refiere a los estándares de calidad a nivel del sistema, con base en valores sociales y paradigmas internacionalmente aceptados y ii) También menciona otra dimensión que es la llamada micro, en la que predominan los estándares de calidad basados en la percepción, sensación, vivencia, satisfacción y situación de salud del usuario.

Uno de los cuestionamientos que surgen sobre el tema corresponde a qué conceptos debieran ser incluidos para determinar "calidad", y en consecuencia, qué debieran ser medidos, donde autores como Massip Pérez et al. identifican los siguientes puntos: confiabilidad, receptividad, competencia, accesibilidad, cortesía, comunicación, credibilidad, seguridad, entender y conocer al cliente y el aspecto tangible del servicio.

Urrutia \& Poupin y Miranda Ortiz et al. resumen los conceptos involucrados en la calidad de atención en dos puntos, constituidos por la persona, usuario o paciente y en un segundo término, el de la calidad del lugar. Estos conceptos están globalmente aceptados y son el objetivo planteado en la mayoría de las encuestas existentes en salud. d'Empaire (2010) establece que la calidad de atención debe enfocar al me- 
nos cuatro aspectos fundamentales: i) Una adecuada prevención y tratamiento, basado en evidencias, el cual debe ser aplicado oportunamente; ii) Deben evitarse o reducirse los posibles daños primarios o secundarios; iii) Se debe respetar la dignidad y derechos de los enfermos y, iv) Debe garantizarse una gestión de recursos eficiente.

Es importante considerar las opiniones de estos autores con la finalidad de llegar a un consenso sobre el concepto de calidad, ya que todos los autores consultados, a su manera, consideran los mismos puntos tratados, sin embargo, éstos reflejan distintas percepciones respecto a importancias de las áreas a considerar en la medición de calidad.

Las instituciones deben ocuparse de la calidad de la atención, la cual debiera ser un imperativo ético; sin embargo, en la mayoría de las situaciones las motivaciones para hacerlo pueden estar fundadas más en el riesgo de acciones legales que en valores positivos (Trucco). Para explicar el concepto de calidad, desde el punto de vista del usuario, se debe analizar el concepto de satisfacción de éste respecto al servicio recibido por parte de la institución demandada.

Según Bucchi et al. (2012), satisfacción se define como la medida en que los profesionales de salud logran cumplir las necesidades y expectativas del usuario. El término satisfacción es muy concurrente en el mundo actual, en la publicidad que nos bombardea en nuestro diario vivir por parte de empresas e instituciones, y que lleva a la sociedad a decidirse muchas veces por un determinado producto o servicio. Velandia Salazar et al. expresan en su artículo que la satisfacción es siempre respecto de algo o de alguien y tiene que ver con algo que se quiere y con lo que se recibe en relación a la espera de un cierto efecto.

Las distintas definiciones nos llevan a cuestionarnos si es posible medir la satisfacción de un usuario, principalmente por la subjetividad que involucra el término. Massip Pérez et al. y Miranda Ortiz et al., por su parte, explican en su artículo que el concepto de satisfacción es subjetivo, que varía entre las personas, y por lo tanto, tiene que ver con las expectativas que se forma alguien de algo. Por su parte, Velandia Salazar et al. y López-Portilla et.al explican que el concepto de satisfacción intenta comunicar sentimientos de favorabilidad o desfavorabilidad, pertenece al campo de las actitudes y, por tanto, del afecto. Esto corrobora la subjetividad del tema, y a su vez, se cuestiona la factibilidad de transformar las distintas sensaciones de los usuarios en un hecho que sea tangible, realizable y practicable. La sociedad, la cultura, y por lo tanto, la relación con otros sujetos, modifican la percepción de satisfacción en las personas.

Según Chang de la Rosa et al. (1999) el concepto de satisfacción en salud incluye tres procesos distintos, entre los que se encuentran los organizativos, la atención recibida y su repercusión en el estado de salud de la población y, por último, el trato recibido durante el proceso de atención por parte del personal. Urrutia \& Poupin explican que los usuarios desean una comunicación más cercana, tanto en tiempo como en la confianza entregada por parte del profesional, que al final, sería uno de los determinantes fundamentales en la satisfacción del paciente.

Trucco y Miranda Ortiz et al. explican que ha aumentado la insatisfacción médica a pesar el éxito de los procedimientos en la historia. Respecto a lo anterior, hay puntos interesantes de analizar, como son el mayor nivel educacional de los pacientes, mayor acceso a la información, universalización de medios de comunicación, costos de atención y a la fragmentación de los profesionales en especialistas de distintas áreas que hacen que el paciente se atienda con varios y en distintos lugares. El profesional ha evolucionado hacia la especialidad en distintas áreas, mejorar en lo específico, descuidando la relación personal con los pacientes.

\section{Ventajas de realizar una encuesta}

Los cambios en la actualidad no son realizados al azar, y la investigación ha tomado un rol predominante en justificar y obtener resultados fruto del método científico, que con argumentación y revisión de distintos autores sumado a los resultados obtenidos en el estudio, permiten realizar cambios sustentados y con un impacto en la sociedad.

Trucco comenta que para realizar una medición de calidad de las instituciones, hay que prestar atención a muchos factores y aplicar múltiples estrategias, incluyendo dimensiones de autorregulación, evaluaciones externas, evaluaciones económicas, desarrollo de la organización y satisfacción de los clientes.

En el actual contexto de la entrega de servicios, la calidad toma un rol protagónico tanto en los países desarrollados como en el nuestro, donde se está apuntando hacia la percepción del usuario como mecanismo de medición de este parámetro, no sólo en las ins- 
tituciones públicas, si no también probadas, tal como explica Tsuchida Fernandez et al. en su artículo. A su vez, Massip Pérez et al. explican que la tendencia actual apunta hacia la humanización de la atención, que a su vez, va directamente relacionado en otorgar servicios de mayor calidad.

En este contexto, para ofrecer servicios de mayor calidad, favoreciendo la creación de instituciones de excelencia, debemos tener instrumentos que constantemente estén aportando datos que muestren la situación actual y, a su vez, que denoten las falencias existentes en el sistema (Massip Pérez et al.). De esta forma es posible realizar las modificaciones a distintos procesos de los distintos ámbitos que involucra la atención, obteniendo una prestación de servicio de mayor calidad (Tsuchida Fernandez et al.) que conlleva en la obtención de satisfacción del paciente usuario del servicio.

A su vez, Riveros \& Berné (2007) y López-Portilla et al. explican que es fundamental la capacidad de gestión de las instituciones que entregan servicios a la población, tanto en capacidades, en compromisos asignados, en la gestión de la información y en la coordinación del servicio, justificando la constante evaluación de estos por personas externas al sistema, en este caso, los usuarios.

Según Velandia Salazar et al., la sociedad espera lograr ciertos estándares de calidad que conlleven a un sistema de salud que sea justo, equitativo, universal, solidario, eficiente, eficaz, democrático, participativo, descentralizado e integrado. Ala vez, este autor expresa que la medición de calidad de atención permite no sólo determinar el desempeño del sistema de salud evaluado, si no determinar cómo es la atención recibida por el paciente, en un contexto de valores, y determinando según los resultados obtenidos la satisfacción o insatisfacción del paciente.

Actualmente muchos sistemas universitarios poseen instancias evaluativas tanto por parte de los alumnados como de los pares, sin embargo, los distintos autores justifican la creación de encuestas de usuarios para lograr mejoras no evidenciadas de los mismos participantes del sistema.

Características del instrumento a crear. Encontramos en la literatura muchos conceptos utilizados en distintos instrumentos existentes principalmente en instituciones de salud, pero que son muy factibles de aplicar.
Una taxonomía con ocho componentes es la propuesta por Ware et al. (citado por Velandia Salazar et al. ), que entre los puntos presenta: i) Aspectos interpersonales: características de la interacción de los prestadores con los pacientes: respeto, cortesía, interés y amistad, entre otros; ii) Calidad técnica de la atención: competencia de los proveedores y adhesión a altos estándares de diagnóstico y tratamiento; iii) Accesibilidad; iv) Aspectos financieros; v) Eficacia y resultados; vi) Continuidad de la atención; vii) Ambiente físico e viii) Integralidad: disponibilidad de recursos para la atención.

Estos ocho aspectos nos dan una visión global de determinada institución, generando la posibilidad de realizar un análisis bastante completo con argumentación sólida, fundamental en la actualidad el lo que respecta al generar cambios.

Trucco y López-Portilla et al. proponen que la medición de resultados incluye al menos tres componentes: i) Medición de resultados: cuantificación sistemática de indicadores de resultados en un punto del tiempo; ii) Monitoreo de resultados: medición repetida de indicadores a lo largo del tiempo y iii) Gestión de resultados: utilización de la información obtenida de la monitorización de indicadores de resultados para mejorar la toma de decisiones clínicas y entrega de servicios.

Al revisar diversos estudios realizados sobre este tema, llama la atención una encuesta creada en torno a un servicio de salud en particular, donde sus autores (Urrutia \& Poupin) y Miranda Ortiz et al. ponen énfasis en ciertos puntos que no deben faltar en un instrumento evaluador de calidad de atención, entre los que incluye: funcionamiento del programa, relación usuario- profesional, infraestructura y recurso humano, educación sobre la patología y, recursos y materiales

\section{DISCUSIÓN}

En la actualidad es evidente la necesidad de generar instrumentos que se utilicen en los variados procesos en los distintos ámbitos de la sociedad, fundamentales en el mundo moderno, donde se observa una fuerte influencia de la globalización, y de la cual nuestro país no está exento.

En este contexto, donde el usuario cumple un rol primordial en el funcionamiento del sistema, nota- 
mos la carencia de métodos de medida que establezcan claramente la situación actual, no sólo de los servicios de salud, si no de clínicas pertenecientes a instituciones universitarias, limita el mejoramiento del servicio entregado tanto para el paciente como para el estudiante, es decir, los usuarios del sistema. Es primordial establecer la problemática evidenciada desde otra mirada, la del usuario del servicio, tal como se está realizando en otros países.

Lo que respecta al concepto de satisfacción lleva directamente a plantear la subjetividad del tema, y que a su vez, cuestiona la factibilidad de transformar las distintas sensaciones de los usuarios en un hecho que sea tangible, realizable y practicable. Sin embargo, y a pesar de lo anteriormente señalado, se debe tratar de plasmar las distintas visiones en datos más objetivos, basados en la investigación científica, la cual justifique de forma adecuada la realización o modificación de los distintos elementos y actores que participan en una institución que se dedica a entregar un servicio.

Muchos autores contemporáneos se basan en los mismos principios y elaboran distintas pautas preocupados de la ya mencionada satisfacción del usuario. Depende del contexto para utilizar estas ideas y adecuarlas a una determinada realidad sociocultural para lograr la creación de un instrumento acorde a las necesidades latentes, y, de esta manera, contribuir a generar cambios positivos en la institución, en este caso educacional (López-Portilla et.al.).

VÁSQUEZ, G.; CORSINI, G.; SILVA, M. FUENTES, J.; CHAHIN, M. \& SANTIBÁÑEZ, J. D. Instrument to measure patient satisfaction about dental care clinic. Int. J. Odontostomat., 10(1):129-134, 2016.

ABSTRACT: This paper discloses the importance of creating an instrument for measuring the satisfaction of attention of the patients attending at the dental clinics of La Frontera University. The criteria used to justify both the creation and the elements that should include the measuring instrument were obtained from a review of several authors, references covering items of various kinds of social, getting data from the current importance of recording and processing user satisfaction as a means of improvement in the modern world the quality of service provided by the institutions. Moreover, delivery components that should be incorporated in a satisfaction survey for the selected target group

KEY WORDS: education, measurement, survey, quality, satisfaction.

\section{REFERENCIAS BIBLIOGRÁFICAS}

Bucchi, C.; Sepúlveda, C.; Monsalves, M. J. \& Bustos, L. Measurement of User Satisfaction in Patients Receiving Emergency Dental Care at Five Primary Health Care Centers. Int. J. Odontostomat., 6(3):275-80, 2012.

Chang de la Rosa, M.; Alemán Lage, M. C.; Cañizares Pérez, M. \& Ibarra, A. M. Satisfacción de los pacientes con la atención médica. Rev. Cuba. Med. Gen. Integral, 15(5):541-7, 1999.

d'Empaire, G. Calidad de atención médica y principios éticos. Acta Bioeth., 16(2):124-32, 2010.

López-Portilla, J. E.; Pilataxi-Sánchez, S.; Rodríguez-Escobar, L. D.; Velásquez-Rivera, A. C.; López-Blandón, M. A.; Martínez-Delgado, C. M. \& Agudelo-Suárez, A. A. Determinantes de la satisfacción de la atención odontológica en un grupo de pacientes atendidos en la Clínica del Adulto de la Facultad de Odontología de la Universidad de Antioquia. Rev. Gerenc. Polit. Salud, 12(24):209-25, 2013.

Massip Pérez, C.; Ortíz Reyes, R. M.; Llantá Abreu, M. C.; Peña Fortes, M. \& Infante Ochoa, I. La evaluación de la satisfacción en salud: un reto a la calidad. Rev. Cuba. Salud Pública, 34(4), 2008.

Ministerio de Salud (MINSAL). Sitio Web del Minsal. Santiago de Chile, Ministerio de Salud, 2013. Disponible en: www.minsal.cl

Miranda Ortiz, J. C.; Guzmán León, R. \& Morales García, M. H. Medición del nivel de satisfacción de la atención estomatológica en las clínicas odontológicas periféricas de la U.J.A.T. Horiz. Sanit., 13(2):207-15, 2014.

Riveros, S. J. \& Berné, M. C. Análisis de la opinión de usuarios sobre calidad percibida y satisfacción con hospitales públicos: Estudio de caso desde la perspectiva de la aplicación del marketing. Rev. Méd. Chile, 135(7):862-70, 2007.

Schweiger, A. L. F. \& Álvarez, D. T. Integración regional, necesidades de salud de la población y dotación de recursos humanos en sistemas y servicios de salud: aproximación al concepto de estimación de brecha. Cad. Saúde Pública, 23(Suppl. 2):S202-13, 2007. 
VÁSQUEZ, G.; CORSINI, G.; SILVA, M. FUENTES, J.; CHAHIN, M. \& SANTIBÁÑEZ, J. D. Instrumento para medir Satisfacción de pacientes sobre atención clínica odontológica. Int. J. Odontostomat., 10(1):129-134, 2016

Trucco, B. M. Ética y calidad en la atención médica y psiquiátrica. Rev. Chil. Neuro-Psiquiatr., 42(2):81-7, 2004.

Tsuchida Fernandez, M. B.; Bandres Sanchez, M. P. \& M. Guevara Linares, X. Nivel de satisfacción general y análisis de la relación médico paciente de los médicos en entrenamiento en las salas de hospitalización de medicina interna. Rev. Med. Hered., 14(4):175-80, 2003.

Urrutia, S. M. T. \& Poupin, B. L. Construcción y validación de un instrumento para medir calidad de atención del programa auge cáncer cervicouterino. Rev. Chil. Obstet. Ginecol., 75(6):396-404, 2010.

Velandia Salazar, F.; Ardón Centeno, N. \& Jara Navarro, M. I. Satisfacción y calidad: análisis de la equivalencia o no de los términos. Rev. Gerenc. Polit. Salud, 6(13):13968, 2007.

\author{
Dirección para Correspondencia: \\ Dr. Gonzalo Vásquez Endara \\ Facultad de Odontología \\ Claro Solar 115 \\ Temuco \\ CHILE
}

Email: gvasquezendara@gmail.com

Recibido: 13-11-2014

Aceptado: 13-04-2016 